\title{
Mixing properties in the advection of passive tracers via recurrences and extreme value theory
}

\author{
Davide Faranda \\ Laboratoire SPHYNX, Service de Physique de l'Etat Condensé, DSM, \\ CEA Saclay, CNRS URA 2464, 91191 Gif-sur-Yvette, Franc£ \\ Xavier Leoncini and Sandro Vaienti \\ Aix Marseille Université, CNRS, CPT, UMR 7332, 13288 Marseille, France and \\ Université de Toulon, CNRS, CPT, UMR 7332, 83957 La Garde, France ${ }^{\dagger}$
}

\begin{abstract}
In this paper we characterize the mixing properties in the advection of passive tracers by exploiting the extreme value theory for dynamical systems. With respect to classical techniques directly related to the Poincaré recurrences analysis, our method provides reliable estimations of the characteristic mixing times and distinguishes between barriers and unstable fixed points. The method is based on a check of convergence for extreme value laws on finite datasets. We define the mixing times in terms of the shortest time intervals such that extremes converge to the asymptotic (known) parameters of the Generalized Extreme Value distribution model. Our technique is suitable for applications in the analysis of other systems where mixing time scales need to be determined and limited datasets are available.
\end{abstract}

\section{INTRODUCTION}

A general purpose of dynamical systems theory is to characterize the stability properties of orbits. The distinction between regular and chaotic dynamics can be easily made in a dissipative case whereas for conservative systems it is usually an hard task especially when we are in presence of many degrees of freedom or for a complex geometry of the phase space. A large number of tools known as indicators of stability have been developed for this purpose; Lyapunov Characteristic Exponents (LCEs) Wolf et al. [1], Rosenstein et al. 22, Skokos [3] and the indicators related to the Return Time Statistics Kac [4], Gao [5], Hu et al. [6], Buric et al. 7] have been used for a long time for such a task. Nevertheless, in the recent past, the need for computing stability properties with faster algorithms and for systems with many degrees of freedom resulted in a renewed interest in the technique and different dynamical indicators have been introduced. The Smaller Alignment Index (SALI) described in Skokos et al. [8] and Skokos et al. 9], the Generalized Alignment Index (GALI), introduced in Skokos et al. [10] and the Mean Exponential Growth factor of Nearby Orbits (MEGNO) discussed in Cincotta et al. [11], Goździewski et al. [12] are suitable to analyze the properties of a single orbit. They are based on the divergence of nearby trajectories and require in principle the knowledge of the exact dynamics. Another class of indicators is based on the round off error properties and has been discussed in Faranda et al. [13]: the divergence between two trajectories starting from the same initial condition but computed with different numeric precision can be used to illustrate the dynamical structure. The so called Reversibility Error that measures the distance between a certain initial condition and the end point of a trajectory iterated forward and backward for the same number of time steps give basically the same information.

These indicators perform generally well when chaotic and regular trajectories are separated. However, in some interesting physical problems, a key question is to distinguish between different mixing regions and possibly recognize the associated mixing time-scales. This problem becomes extremely relevant for describing the dynamics of a passive tracer in an array of alternating vortices. This type of flows emerge from Rayleigh-Bénard convection and can be studied as well experimentally using electromagnetic forces [14-16. One of the primary interest in this type of flow is that it can be generated by different instabilities, and as such it lays on one of the paths to turbulence. When considering mixing or transport in fluids, one usually rely more on a Lagrangian than a Euler point of view. As such regarding transport, the dynamics of passive tracers advected in a two dimensional incompressible flow is Hamiltonian. In this setting the canonical variables are directly the space ones, allowing for a direct visualization of the phase space. Moreover we have an accessible framework to test theoretical ideas and have a direct grasp of their physical consequences.

In our study we consider the flows proposed in Benzekri et al. [17, Bachelard et al. [18]. These flows theoretically

*Electronic address: davide.faranda@cea.fr

${ }^{\dagger}$ Electronic address: Xavier.Leoncini@cpt.univ-mrs.fr 
offer the peculiarity of "targeted mixing", meaning that mixing is efficiently performed within cells formed by one dimensional barriers. This offers a suitable setting to test our approach and quantify it versus the already proposed measurements. Before moving one, we give a brief description of the flows. We first start with the integrable stream function that describes an array of alternating vortices .

$$
\Psi_{0}(x, y)=\alpha \sin x \sin y
$$

Here the $x$-direction is the one along the channel and the $y$-direction is the bounded orthogonal one. The amplitude $\alpha$ sets the maximal value the velocity. The dynamics resulting from the Hamiltonian which is identical to the stream function Eq. (1) is integrable and thus no chaotic mixing occurs. In this setting, tracers motion are confined within barriers delimited by the invariant lines $y=\pi$ and $y=0$ and the other invariant lines which are localized at $x=m \pi$ for $m \in \mathbb{Z}$ along the channel. As we shall see later, it is important to mention that these points are joined by vertical heteroclinic connections for which the stable and unstable manifolds coincide. The phase space, which is here the real space, is then characterized by a channel populated by alternating vortices with separatrices localized at $x=m \pi$ for $m \in \mathbb{Z}$.

In order to visualize the phenomenon of chaotic advection in experiments [14-16, a typical perturbation $f(x, y, t)$ is introduced as a time dependent forcing. This allows for instance to subsequently study the transport and mixing properties. To be more explicit, the perturbation corresponds to a modification of the stream function :

$$
\Psi_{c}(x, y, t)=\Psi_{0}(x+f(x, y, t), y) .
$$

For instance, in the experiment a flow has been realized which is well modeled by the following stream function [14]

$$
\Psi_{1}(x, y, t)=\alpha \sin \left(x+\epsilon \sin \omega_{0} t\right) \sin y .
$$

The perturbation $f$ becomes simply $f=\epsilon \sin \omega_{0} t$ and describes the lateral oscillations of the roll patterns where $\epsilon$ and $\omega_{0}$ are respectively the amplitude and the angular frequency of the lateral oscillations. Setting the proper time units, we may assume that $\omega_{0}=1$. In this setting, the field lines have not changed but simply oscillate back and forth along the channel, and chaotic advection is triggered. This perturbation breaks the separatrices and invariant tori, leading to chaotic mixing along the channel. However we still have invariant tori corresponding to stable island of regular motion near the vortex cores. These act as transport barrier and mixing is not uniform. Regarding this problem a different perturbation was proposed in Benzekri et al. [17, Bachelard et al. [18]. This perturbation allows in some windows of parameters to only preserve the separatrices while destroying all regular tori. The separatrices acting as transport barrier we end up with a homogeneous mixing within cells delimited by the separatrices. The proposed perturbation writes :

$$
\Psi_{c}(x, y, t)=\alpha \sin \left[x+\epsilon \sin t+\alpha \cos y C_{\epsilon}(t)\right] \sin y
$$

where

$$
C_{\epsilon}(t)=\sum_{n \geq 0} \frac{-2}{2 n+1} \mathcal{J}_{2 n+1}(\epsilon) \cos (2 n+1) t
$$

and $\mathcal{J}_{l}($ for $l \in \mathbb{N}$ ) are Bessel functions of the first kind.

We defer to Bachelard et al. [18] for the details but we recall that this perturbation has two main purposes: i) particles remain trapped within a specific domain bounded by two oscillating barriers (suppression of chaotic transport along the channel), ii) the stochastic sea seems to cover the whole domain (enhancement of mixing within the cells).

In Benzekri et al. [17, Bachelard et al. [18, the mixing properties of the barriers have been analyzed for several specific values of $\alpha, \epsilon$. For instance the finite time Lyapunov map was computed, showing some kind of uniform mixing. When performing an analysis using Poincaré recurrences and a finite time average recurrence time, the barriers naturally emerged however no remarkable differences between the barriers and the fixed points stood out.

In this paper we try to overcome this difficulty by suggesting a new method for the characterization of mixing times based on the Extreme Value Theory (EVT) for dynamical systems. In particular, we will show that by exploiting the EVT, one is able to observe differences between unstable fixed points and barriers as well as to extrapolate the characteristic time scales such that the dynamics become mixing.

The EVT was originally introduced by Fisher and Tippett [19], Gnedenko [20] to study the maxima of a series of independent and identical distributed variables: under very general hypothesis a limiting distribution called Generalized Extreme Value (GEV) distribution exists for the series of extremes. An extensive account of recent results and relevant applications is given in Ghil et al [21]. 
In the recent past the EVT has been adapted to study the output of dynamical systems. As we will explain in detail in the next section, it is not trivial to observe asymptotic GEV distributions in dynamical systems: some sort of independence of maxima must be recovered by requiring certain mixing conditions on the orbits. Furthermore, we need to introduce some peculiar observables that satisfy the condition proposed by Gnedenko 20] on the parent distribution of data: they are related to the closest return of a trajectory in a ball centered around a reference point of the attractor and therefore allow a very detailed description of the dynamics in the neighborhood of the chosen point.

The parameters of the distribution are dependent on the geometrical properties of the system i.e. the local dimension of the attractor Freitas et al. 22, Lucarini et al. 23]. When this properties are known, like for the advection of the passive tracers, one can study the convergence of finite datasets to the asymptotic parameters recovered in the limit of infinitely long time series. For finite datasets, the rate of convergence will be directly related to the chosen point of the phase space and to the local mixing structure of the trajectories passing nearby. The main idea is to define a mixing time scale based on the minimum time interval for the selection of maxima which allows for recovering the asymptotic parameters predicted by the theory. We will tackle this problem in the remaining of the paper which is organized as follows: in Section 2 we recall the results of EVT for dynamical systems and explain the numerical algorithm and procedures used to compute the parameters of the GEV distribution introduced in Faranda et al. [24]. In Section 3 we describe the model and the numerical results. Section 4 is dedicated to discussion and possible outlooks

\section{ASYMPTOTIC EXTREME VALUE THEORY FOR DYNAMICAL SYSTEMS}

Gnedenko [20] studied the convergence of maxima of i.i.d. variables

$$
X_{0}, X_{1}, . . X_{m-1}
$$

with cumulative distribution function (cdf) $F(x)$ of the form:

$$
F(x)=P\left\{a_{m}\left(M_{m}-b_{m}\right) \leq x\right\}
$$

where $a_{m}$ and $b_{m}$ are normalizing sequences and $M_{m}=\max \left\{X_{0}, X_{1}, \ldots, X_{m-1}\right\}$. Eq. 6 can be rewritten as $F\left(u_{m}\right)=$ $P\left\{M_{m} \leq u_{m}\right\}$ where $u_{m}=x / a_{m}+b_{m}$. Under general hypothesis on the nature of the parent distribution of data, the cdf of maxima $F(x)$ converges to a single family of generalized distribution called GEV distribution with cdf:

$$
F_{G}(x ; \mu, \sigma, \kappa)=\exp \left\{-\left[1+\kappa\left(\frac{x-\mu}{\sigma}\right)\right]^{-1 / \kappa}\right\}
$$

which holds for $1+\kappa(x-\mu) / \sigma>0$, using $\mu \in \mathbb{R}$ (location parameter) and $\sigma>0$ (scale parameter) as scaling constants in place of $b_{m}$, and $a_{m}$ [25. In particular, in Faranda et al. [26] we have shown that the following relations hold:

$$
\mu=b_{m} \quad \sigma=\frac{1}{a_{m}} .
$$

$\kappa \in \mathbb{R}$ is the shape parameter also called the tail index and discriminate the type of classical extreme value laws: when $\kappa \rightarrow 0$, the distribution corresponds to a Gumbel type ( Type 1 distribution). When $\kappa$ is positive, it corresponds to a Fréchet (Type 2 distribution); when $\kappa$ is negative, the extreme value law corresponds to a Weibull (Type 3 distribution).

In the last decade many works focused on the possibility of treating time series of observables of deterministic dynamical system using EVT. The first rigorous mathematical approach to extreme value theory in dynamical systems goes back to the pioneer paper by Collet [27]. Important contributions have successively been given in Freitas and Freitas [28], Freitas et al. [22, Freitas et al. [29] and by Gupta et al. [30. The goal of all these investigations was to associate to the stationary stochastic process given by the dynamical system, a new stationary independent sequence: when the latter sequence satisfies one of the classical three extreme value laws, the same result also holds for the original dynamical sequence. We summarise shortly the main findings of the theory.

Let us consider a dynamical systems $(\Omega, \mathcal{B}, \nu, f)$, where $\Omega$ is the invariant set in some manifold, usually $\mathbb{R}^{d}, \mathcal{B}$ is the Borel $\sigma$-algebra, $f: \Omega \rightarrow \Omega$ is a measurable map and $\nu$ a probability $f$-invariant Borel measure. 
In order to adapt the extreme value theory to dynamical systems, we introduce the stationary stochastic process $X_{0}, X_{1}, \ldots$ given by:

$$
X_{m}(x)=g\left(\operatorname{dist}\left(f^{m}(x), \zeta\right)\right) \quad \forall m \in \mathbb{N},
$$

where 'dist' is a distance on the ambient space $\Omega, \zeta$ is a given point and $g$ is an observable function. The probability measure is here the relevant invariant measure $\nu$ for the dynamical system often called the physical measure. Hereinafter we will use three types of observables $g_{i}, i=1,2,3$ that are suitable to obtain one of the three types of extreme value laws for normalized maxima:

$$
\begin{gathered}
g_{1}(x)=-\log (\operatorname{dist}(x, \zeta)), \\
g_{2}(x)=\operatorname{dist}(x, \zeta)^{-1 / \beta}, \\
g_{3}(x)=C-\operatorname{dist}(x, \zeta)^{1 / \beta},
\end{gathered}
$$

where $C$ is a constant and $\alpha>0 \in \mathbb{R}$ Collet [27], Freitas et al. [22].

By using these observables we get convergence to the Type 1, 2, or 3 distribution if one can prove two sufficient conditions called $D_{2}$ and $D^{\prime}$ which basically require a sort of independence of the stochastic dynamical sequence in terms of uniform mixing conditions on the distribution functions. In particular condition $D_{2}$, introduced in its actual form in Freitas and Freitas [28], could be checked directly by estimating the rate of decay of correlations for a suitable class of observables. We summarize these conditions as follows:

If $X_{m}, m \geq 0$ is our stochastic process, we can define $M_{j, l} \equiv \max \left\{X_{j}, X_{j+1}, \cdots, X_{j+l}\right\}$ and set $M_{0, m}=M_{m}$. The condition $D_{2}\left(u_{m}\right)$ holds for the sequence $X_{m}$ if for any integer $l, t, m$ we have

$$
\left|\nu\left(X_{0}>u_{m}, M_{t, l} \leq u_{m}\right)-\nu\left(X_{0}>u_{m}\right) \nu\left(M_{t, l} \leq u_{m}\right)\right| \leq \gamma(m, t),
$$

where $\gamma(m, t)$ is non-increasing in $t$ for each $m$ and $m \gamma\left(m, t_{m}\right) \rightarrow 0$ as $m \rightarrow \infty$ for some sequence $t_{m}=o(m), t_{m} \rightarrow \infty$. Let $\left(k_{n}\right)_{n \in \mathbb{N}}$ be a sequence of integers such that

$$
k_{n} \rightarrow \infty \text { and } k_{n} t_{n}=o(n) .
$$

We say that $D^{\prime}\left(u_{n}\right)$ if there exists a sequence $\left\{k_{n}\right\}_{n \in \mathbb{N}}$ satisfying 12 and such that

$$
\lim _{n \rightarrow \infty} n \sum_{j=1}^{\left\lfloor n / k_{n}\right\rfloor} \mathbb{P}\left(X_{0}>u_{n}, X_{j}>u_{n}\right)=0 .
$$

Here $\lfloor m / l\rfloor$ indicates the integer part of $m / l$.

Instead of checking the previous conditions, we can use other results that established a connection between the extreme value laws and the statistics of first return and the Hitting time statistics (hereinafter HTS) [22, 31. Before introduing the HTS, we need first to define the recurrence time $\tau_{A}$ in a measurable set $A \in \Omega$, as

$$
\tau_{A}(x)=\inf _{t \geq 1}\left\{x \in A: f^{t}(x) \in A\right\}
$$

and the average recurrence time $\left\langle\tau_{A}>\right.$ as

$$
<\tau_{A}>=\int \tau_{A}(x) \mathrm{d} \mu_{A}(x) \quad \mu_{A}(B)=\frac{\mu(A \cap B)}{\mu(A)} .
$$

We notice that, whenever the measure $\mu$ is ergodic, Kac'theorems ensures that $\left\langle\tau_{A}\right\rangle=\mu(A)^{-1}$. Following Hirata et al. 32] and Buric et al. [33, we define the HTS as the following limit (whenever it exists): 


$$
H(t)=\lim _{\mu(A) \rightarrow 0} \mu_{A}\left(A_{>t}\right) \quad A_{>t} \equiv\left\{x \in A: \frac{\tau_{A}(x)}{<\tau_{A}>}>t\right\} .
$$

In particular, Freitas et al. 22] and Freitas et al. 31] showed that for dynamical systems preserving an absolutely continuous invariant measure or a singular continuous invariant measure $\nu$, the existence of an exponential HTS on balls around almost any point $\zeta$, namely $H(t)=e^{-t}$, implies the existence of extreme value laws for one of the observables of type $g_{i}, i=1,2,3$ described above. The converse is also true, namely if we have an extreme value law which applies to the observables of type $g_{i}, i=1,2,3$ achieving a maximum at $\zeta$, then we have exponential HTS to balls with center $\zeta$. Recently, these results have been generalized to local returns around balls centered at periodic points Freitas et al. 29] and for stochastically perturbed dynamical systems [34 36.

\section{THE METHOD FOR FINITE TIME DATASETS}

In Faranda et al. 26] and Lucarini et al. 23, the authors have analyzed both from an analytical and a numerical point of view the Extreme Value distribution in a wide class of low dimensional maps showing that, when the conditions $D^{\prime}$ and $D_{2}$ are verified, the block maxima approach can be used to study extrema. This approach consists of dividing the data series of length $k$ of some observable into $n$ bins each containing the same number $m$ of observations, and selecting the maximum (or the minimum) value in each of them 37. The GEV distribution is obtained by performing a fit of the histogram of maxima (minima) to the GEV model. When one uses the $g_{i}$ observable functions and the underlying dynamic is mixing, the asymptotic GEV parameters are known and depend on $m$ (or equivalently $n$ ) and the local dimension of the attractor $d$. In particular, the following equations hold:

For $g_{1}$ type observable:

$$
\sigma=\frac{1}{d} \quad \mu \sim \frac{1}{d} \ln (k / n) \quad \kappa=0
$$

For $g_{2}$ type observable:

$$
\sigma \sim n^{-1 /(\alpha d)} \quad \mu \sim n^{-1 /(\alpha d)} \quad \kappa=\frac{1}{\beta d}
$$

For $g_{3}$ type observable:

$$
\sigma \sim n^{1 /(\alpha d)} \quad \mu=C \quad \kappa=-\frac{1}{\beta d}
$$

Here $\sim$ means asymptotically for $m, n \rightarrow \infty$. At finite time, the convergence depends on the rate of mixing around the point $\zeta$. We can have one of the following behavior:

- For $\zeta$ s on periodic or quasi-periodic orbits we do not observe convergence to the GEV distribution. If the motion is purely periodic, the asymptotic extreme value law is a Dirac's delta otherwise it is a collection of Heaveside functions modulated by the shape of the $g_{i}$ s.

- For $\zeta$ s on mixing orbits there exists a value of $m$ such that the previous Eqs. 15 17 hold. This value of $m$ can be defined as the shortest mixing time scale. As we will see from the numerical analysis, detailed mixing properties of the phase space may be explored with this method.

- For $\zeta$ s located in the proximity of unstable fixed points, the previous Eqs 15, 17 do not hold. Extremes cluster around the fixed points making the convergence as slower as closer we get to the fixed point.

\section{A. A practical numerical algorithm}

In Faranda et al. [24] we have introduced a simple algorithm to get the parameter specified in Eqs 15.17 
1. Compute the orbit of the dynamical system for $k$ iterations.

2. Compute the series $X_{m}(x)=g\left(\operatorname{dist}\left(f^{m}(x), \zeta\right)\right)$ where $\zeta$ is a point of the phase space.

3. Divide the series in $n$ bins each containing $m$ data.

4. Take the maximum in each bin and fit the GEV distribution.

For the inference, we have used the Maximum Likelihood Estimation (MLE) procedure explained in Faranda et al. [24]. There the authors introduced the method and tested it on the relevant example of the Standard map. They characterized different regions of the phase space in terms of rate of convergence to the parameters expected by Eqs. 15. 17. The results have been checked against the Divergence of two nearby trajectories and the Reversibility error as introduced in Faranda et al. [13. Once the experimental parameters $\mu, \sigma, \kappa$ are obtained by a fit at a certain $m$, there are only two possibilities:

- If the fit succeeds one can repeat the experiment for shorter bin lengths and find the smallest $m$ such that, for the chosen $\zeta$, the fit converges. This defines the shortest mixing time scale.

- If the fit fails one should repeat the experiment by increasing the size of $m$ until it is possible to retain a sufficient number of maxima to perform a reliable fit to the GEV model.

As we have already said, for purely periodic orbit one never finds a $m$ such that the fit converges. In the next section we show how the application of this method provides reliable results in the case of the advection of a passive tracer.

\section{RESULTS}

We present the results obtained for the Hamiltonian dynamics associated to the stream function in Eq. 4 . By definition, the advection term relates to the action of being moved by and with a flow. The velocity field is then obtained by $\mathbf{v}=\operatorname{curl}(\psi \hat{\mathbf{z}})$, where $\hat{\mathbf{z}}$ is the unit vector normal to the flow. The flow of passive tracers exhibits a Hamiltonian structure :

$$
\dot{x}=-\frac{\partial \Psi}{\partial y}, \quad \dot{y}=\frac{\partial \Psi}{\partial x}
$$

where $(x, y)$ corresponds to the coordinates of the tracer on the plane. The space variables $(x, y)$ are canonically conjugate for the stream function $\Psi$ which acts as the Hamiltonian of the system. Hence the phase space is formally the two dimensional physical space (with the addition of time).

In Fig. 1 two Poincaré section obtained by numerical In Fig. 1 two Poincaré sections obtained by numerical have been performed by setting the time step $\Delta T=5 \cdot 10^{-3}$, and computing the trajectory of 1000 particles, released at $x_{0}=3.3, y_{0}=1.6$ for 1000 time iterations. The left panel of Fig 1 refers to the set of parameters $\alpha=1, \epsilon=0.63$, the right one to $\alpha=1, \omega=0.8$. For the first set of parameters, stability islands are clearly visible in the domain whereas for $\alpha=1, \omega=0.8$, the domain looks well mixed on the time scale considered.

For the extreme value analysis we consider the experiment with $\epsilon=0.63$. First one can check that the method based on the EVT allows for recognizing the different stability regions. The experiment follows the set up described before: we consider a very long run - $s=10^{6}$ iterations - and take 2000 different $\zeta$ points uniformly distributed in the domain at which the extreme value statistics for the observables $g_{i}$ is computed by taking $m=250, n=4000$ maxima. The results obtained with the EVT analysis are qualitatively similar for the three observables considered so that in Fig. 2 we have chosen to represent only the results for the observable $g_{3}$ with $\beta=3$. Since we are dealing with a bi-dimensional system, we expect to find $\xi=-1 / 6$ in the chaotic region. This is true in a wide region of the phase space which can be identified as a region where orbits mix efficiently. However we observe a different behavior in correspondence of the regular islands where divergence from the theoretical expected parameters are observed. In Fig. 2 the locations of the barriers is well highlighted as it forms a sort of frame of divergent values of $\kappa$ around the figure (red areas). Within the barriers the values of the shape parameters are slightly different than in the chaotic sea and points to regions where intermediate properties between the regular islands and the chaotic sea are present.

In order to better quantify the effect of the barriers, we have isolated a region of the phase space corresponding to the left border of the plot and repeated the analysis. The results are presented in Fig. 3 for $g_{1}$ (left) and $g_{3}$ (right). The expected values in the chaotic see are respectively 0 and $-1 / 6$ and they correspond to the highest values of the color-bars. The position of the barriers and the in-homogeneities within them are evident. In particular, one can recognize the fixed points (top left) and (bottom right) for the remarkable deviations from the theoretical parameters. 

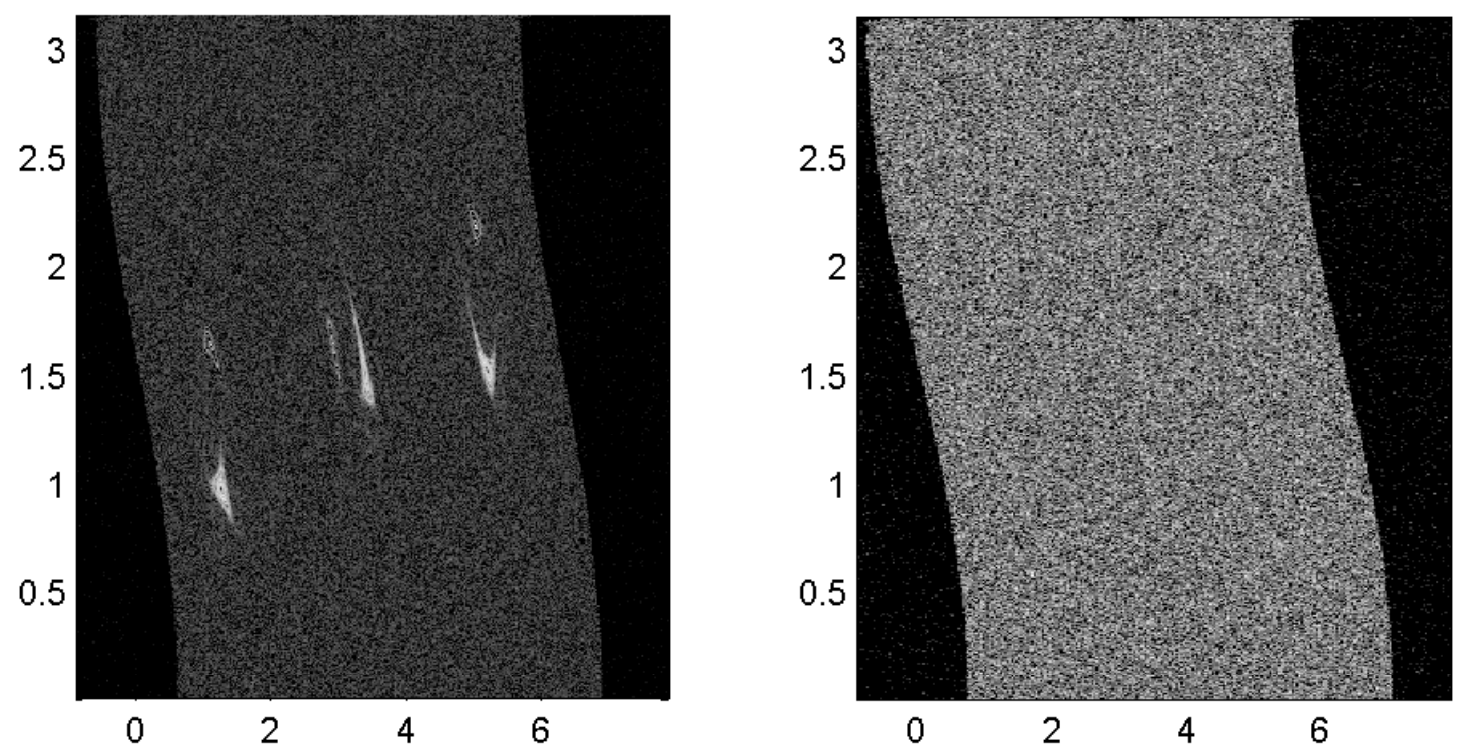

Figure 1: Poincaré sections for the stream function in Eq. 4. The parameters are $\alpha=1, \epsilon=0.63$ (left) and alpha=1, $\epsilon=0.8$ (right).

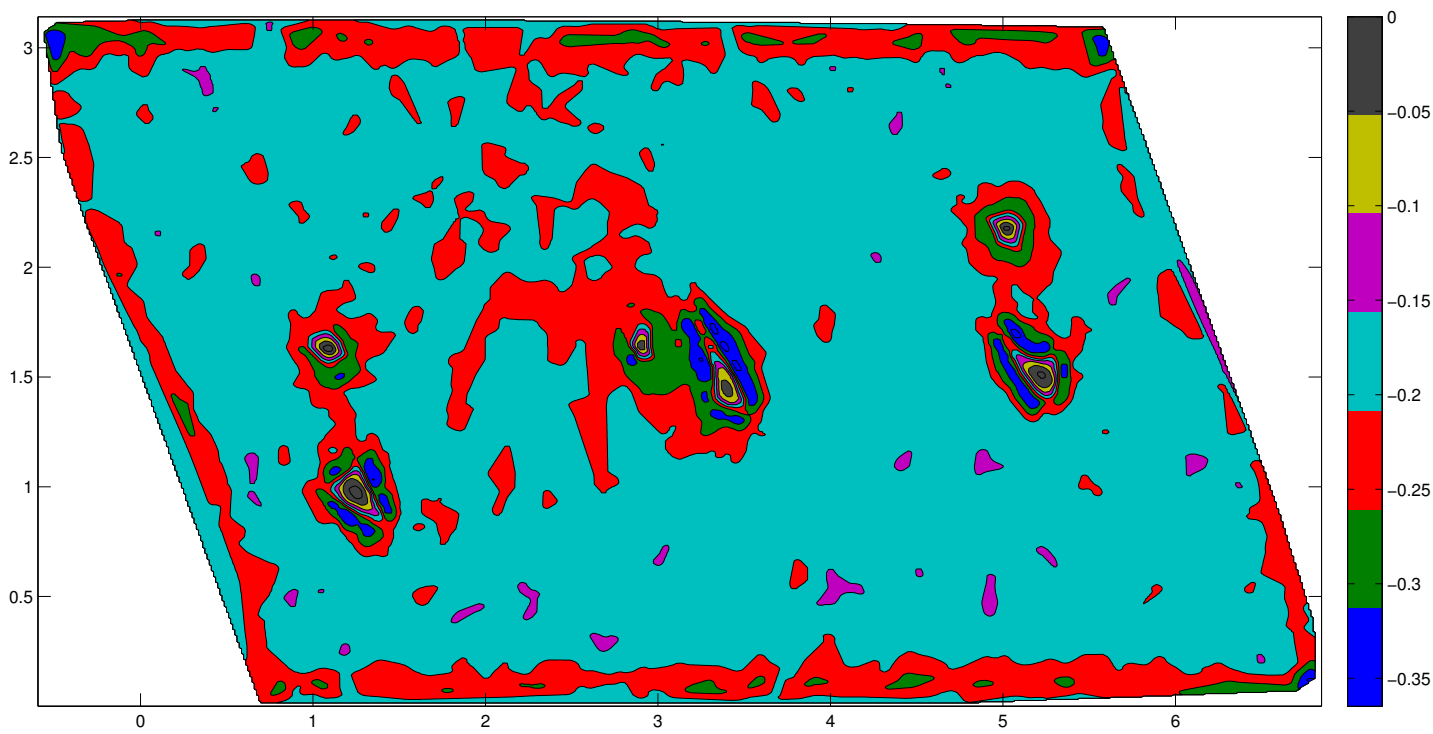

Figure 2: $\kappa$ for the observable $g_{3}, \beta=3$ for an ensemble of 2000 points. The theoretical expected value is $\kappa=-1 / 6$ (azure). See text for a description.

Other methods (i.e. the Poincaré recurrences technique applied in Bachelard et al. [18) do not distinguish between barriers and fixed points; actually the latter method quantify the frequency of visits in small balls spread in the phase space. Instead the parameters of the EVT depend on the structure of the fixed points via the so called extremal index $\theta$, introduced by Freitas et al. [29] and further analyzed by Faranda et al. 35. By invoking again the equivalence between extreme value statistics and statistics of return times, the presence of an extremal index simply means that the statistics of the first return time in a ball shrinking to zero around a periodic point of (minimal) period $p$ and normalized with the average recurrence time, converge to $e^{-t \theta}$, where $\theta:=\phi(p)$ is a non-linear function $\phi$ of the 

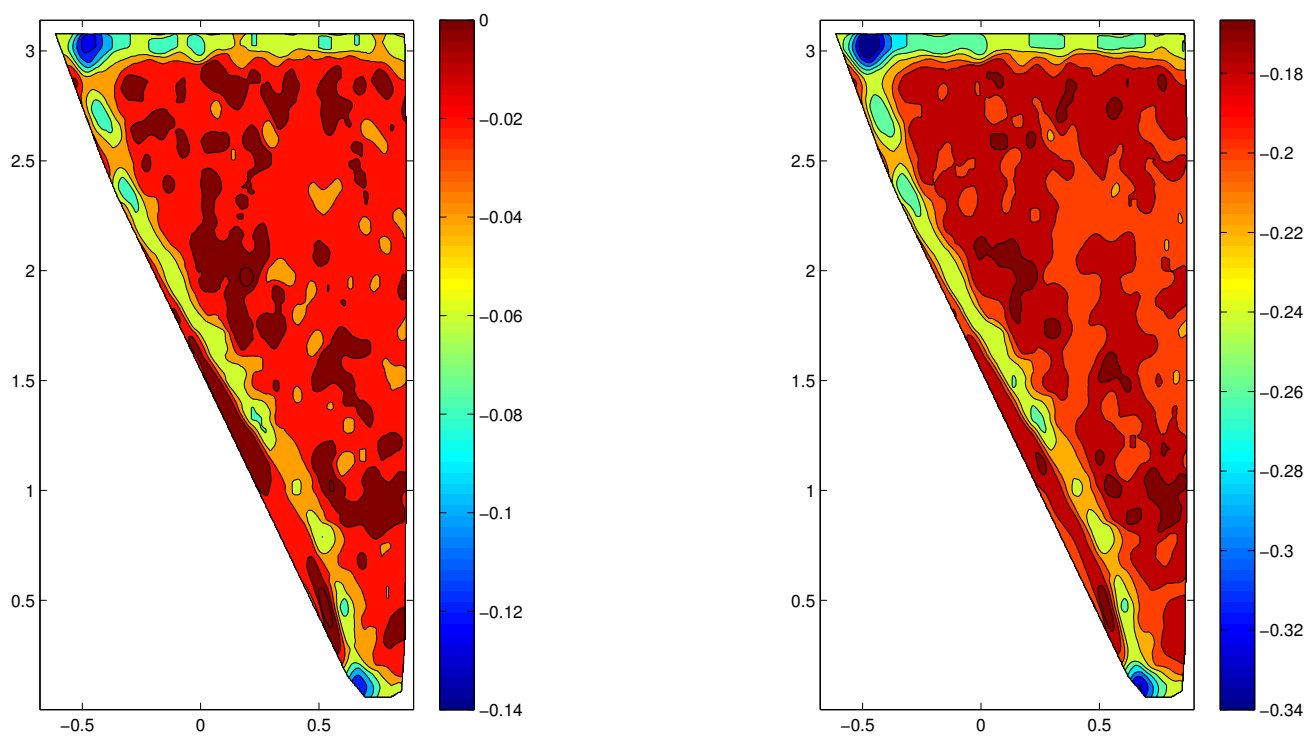

Figure 3: Left: $\kappa$ for the observable $g_{1}$. Right: $\kappa$ for the observable $g_{3}, \beta=3$. Ensemble of 2000 points. The theoretical expected value are $\kappa=0$ for $g_{1}$ and $\kappa=-1 / 6$ for $g_{3}$. See text for a description.

period $p$ determined by the potential associated to the invariant measure $\mu$ 38. Instead the distribution is simply the exponential one $e^{-t}$ around a non-periodic point discussed after Eq. 14

This divergence between the behavior around periodic and non-periodic points reflects in a departure of the theoretical parameters expected for the GEV in the regime of pure Gumbel's law shown in Fig. 3.

This qualitative considerations can be made quantitative when the bin length is varied. This way, one can reliably compute the time scale on which the barriers become mixing. In order to do so, we consider three different ensembles of 500 points each. The first one contains all points extracted in the periodic sea, the second one includes only points located on the barriers and the third one points in a neighborhood of the fixed point. For each ensemble, we computed the average shape parameter $\kappa$ at several bin lengths. The results are displayed in Fig. 4 together with a linear fit of the data. For the chaotic points, no substantial dependence on the $m$ chosen is visible and the value are substantially distributed around the expected value $\kappa=0$. The points located in the barriers show divergent values of the parameters and, for increasing $m$, approach a fully chaotic behavior extrapolated at $m=10000$. The shape parameter is highly divergent in the neighborhood of the fixed points and two different linear approximation have been computed. Note that, if exactly the fixed point is considered, the fit does not improve even at higher $m$ but oscillates on negative values. The explanation for the direction of the drift (towards more negative values of $\kappa$ ) follows the argument described in Faranda et al. [39.

\section{FINAL REMARKS}

In this paper we have defined a rigorous approach for the definition of mixing time scales by exploiting the results of the extreme value theory for dynamical systems. With respect to classical methods based on the Poincaré recurrences theory, our method is able to discriminate between slow mixing regions - the barriers and the fixed points of the dynamics. Previous analysis (see Bachelard et al. [18] ) could not highlight any difference between fixed points and barriers by using the statistics of Poincaré recurrences. The effectiveness of our method is based on the fact that the asymptotic statistics can be computed analytically just by knowing the geometrical properties of the system (the local dimensions). Once the asymptotic parameters are known, a mixing time is intuitively defined as the minimum bin length of the block-maxima approach such that one obtains convergence to the predicted extreme value laws. Differences between barriers and fixed points appear because asymptotic laws are strongly modified in the proximity of unstable fixed points by the existence of a regular dynamics which is responsible for the clustering of extreme events. Clusters introduce an extra parameter in the theory, the so-called extremal index, which we used indirectly for discriminating between fixed points and barriers. 


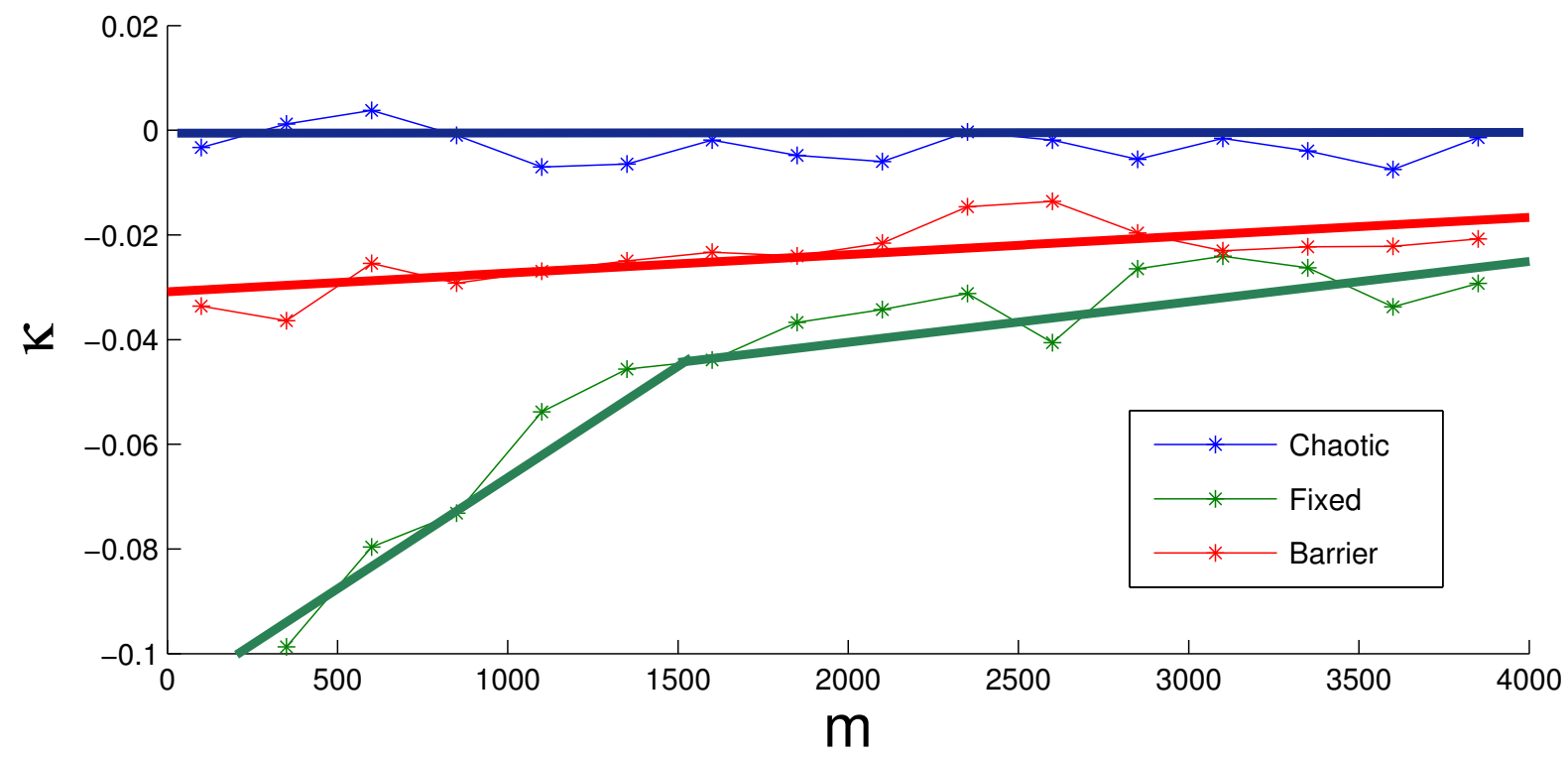

Figure 4: $\quad \kappa$ for the observable $g_{1}$ at different bin lengths $m$ for three different ensemble of points. The theoretical expected values is $\kappa=0$ (black line). See text for a description.

The results obtained in this paper can be extended to a large class of systems where the computation of mixing time scales are of any interest. Moreover, one has a powerful tool to study the dynamics around unstable fixed points. In a future publication we will address the issue of having an extremal index different than one. In particular, this implies an interesting non-equivalence between the block-maxima approach discussed in the present paper and the peak-over-threshold approach.

Other extensions of our methods concern the applicability on geophysical flows. An example is given in [36. There, mixing-time scales are linked to the definition of normal or extreme recurrences of air temperature data. It will be interesting to apply the findings of this paper to extend the results presented in [36] and, by including other atmospheric variables, construct a more complex geography of the phase space.

\section{ACKNOWLEDGMENTS}

SV was supported by the ANR- Project Perturbations, by the PICS ( Projet International de Coopération Scientifique), Propriétés statistiques des systémes dynamiques déterministes et aléatoires, with the University of Houston, n. PICS05968 and by the projet MODE TER COM supported by Region PACA, France. SV and DF acknowledge the Newton Institute in Cambridge where this work was completed during the program Mathematics for the Fluid Earth. DF acknowledges the support of a CNRS post-doctoral grant.

[1] A. Wolf, J. B. Swift, H. L. Swinney, and J. A. Vastano, Physica D 16, 285 (1985).

[2] M. T. Rosenstein, J. J. Collins, and C. J. De Luca, Physica D 65, 117 (1993).

[3] C. Skokos, in Lect Notes Phys, Berlin Springer Verlag, Vol. 790 (2010) pp. 63-135.

[4] M. Kac, Proc Nat Acad Sci USA 20, 376 (1934).

[5] J. B. Gao, Phys Rev Lett 83, 3178 (1999).

[6] H. Hu, A. Rampioni, L. Rossi, G. Turchetti, and S. Vaienti, Chaos 14, 160 (2004).

[7] N. Buric, A. Rampioni, and G. Turchetti, Chaos Soliton Fract 23, 1829 (2005).

[8] C. Skokos, C. Antonopoulos, T. Bountis, and M. Vrahatis, in Proc of 4th GRACM Congress on Computational Mechanics, Vol. 4 (Citeseer, 2002).

[9] C. Skokos, C. Antonopoulos, T. C. Bountis, and M. Vrahatis, J Phys A-Math Gen 37, 6269 (2004).

[10] C. Skokos, T. C. Bountis, and C. Antonopoulos, Physica D 231, 30 (2007). 
[11] P. M. Cincotta, C. M. Giordano, and C. Simó, Physica D 182, 151 (2003).

[12] K. Goździewski, E. Bois, A. J. Maciejewski, and L. Kiseleva-Eggleton, Astron Astrophys 378, 569 (2001).

[13] D. Faranda, M. F. Mestre, and G. Turchetti, International Journal of Bifurcation and Chaos 22 (2012).

[14] T. Solomon and J. Gollub, Physical Review A 38, 6280 (1988).

[15] T. H. Solomon, N. S. Miller, C. J. Spohn, and J. P. Moeur, in AIP Conference Proceedings, Vol. 676 (2003) p. 195.

[16] H. Willaime, O. Cardoso, and P. Tabeling, Physical Review E 48, 288 (1993).

[17] T. Benzekri, C. Chandre, X. Leoncini, R. Lima, and M. Vittot, Physical review letters 96, 124503 (2006).

[18] R. Bachelard, T. Benzekri, C. Chandre, X. Leoncini, and M. Vittot, Physical Review E 76, 046217 (2007).

[19] R. Fisher and L. Tippett, in Proceedings of the Cambridge philosophical society, Vol. 24 (1928) p. 180.

[20] B. Gnedenko, Ann Math 44, 423 (1943).

[21] M. Ghil et al, Nonlinear Process Geophys 18, 295 (2011).

[22] A. Freitas, J. Freitas, and M. Todd, Probab Theory Relat Fields , 1 (2009).

[23] V. Lucarini, D. Faranda, G. Turchetti, and S. Vaienti, Chaos: An Interdisciplinary Journal of Nonlinear Science 22, 023135 (2012).

[24] D. Faranda, V. Lucarini, G. Turchetti, and S. Vaienti, International Journal of Bifurcation and Chaos 22 (2012).

[25] J. Pickands III, Ann Math Stat 39, 881 (1968).

[26] D. Faranda, V. Lucarini, G. Turchetti, and S. Vaienti, J Stat Phys , 1 (2011).

[27] P. Collet, Ergod Theor Dyn Syst 21, 401 (2001).

[28] A. Freitas and J. Freitas, Stat Probab Lett 78, 1088 (2008).

[29] A. C. M. Freitas, J. M. Freitas, and M. Todd, Advances in Mathematics 231, 2626 (2012).

[30] C. Gupta, M. Holland, and M. Nicol, Ergodic Theory and Dynamical Systems 31, 1363 (2011).

[31] A. Freitas, J. Freitas, and M. Todd, J Stat Phys , 1 (2011).

[32] M. Hirata, B. Saussol, and S. Vaienti, Comm Math Phys 206, 33 (1999).

[33] N. Buric, A. Rampioni, G. Turchetti, and S. Vaienti, J Phys A-MathGen 36, L209 (2003).

[34] D. Faranda and S. Vaienti, arXiv preprint arXiv:13085624 (2013).

[35] D. Faranda, J. Freitas, V. Lucarini, G. Turchetti, and S. Vaienti, Nonlinearity 26, 2597 (2013).

[36] D. Faranda and S. Vaienti, Geophysical Research Letters 40, 5782 (2013).

[37] S. Coles, J. Heffernan, and J. Tawn, Extremes 2, 339 (1999).

[38] N. Haydn and S. Vaienti, Probability theory and related fields 144, 517 (2009).

[39] D. Faranda, V. Lucarini, and P. Manneville, To appear: Chaos, Solitons and Fractals (2014). 